\title{
Evolution of the diffusion layer in a CMSX4 single crystal superalloy
}

\author{
Elisabetta Gariboldi ${ }^{1,}$, Xinghua Han ${ }^{1, b}$, Giulioantonio Longo ${ }^{2, c}$ \\ and Giovanni Paolo Zanon ${ }^{3, d}$ \\ ${ }^{1}$ Politecnico di Milano, Dipartimento di Meccanica, Via La Masa 1, 20156 Milano (Italy) \\ ${ }^{2}$ Avio SpA, viale Impero snc, 80030 Pomigliano d'Arco (Italy) \\ ${ }^{3}$ Avio SpA, via I Maggio 56, 10040 Rivalta di Torino (Italy)

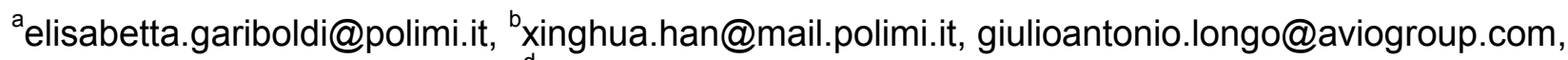 \\ dgiovanni.zanon@aviogroup.com
}

Key words: CMSX4 alloy, aluminizing, anistropy

\begin{abstract}
Aluminising processes are well-known techniques industrially adopted to enrich of aluminium the surface layers of Ni-based alloys thus improving their resistance to environmental interaction at high-temperature. The results of aluminising processes are typically described in terms of the presence, compositions and thickness of the sequence of layers at the surface of the treated parts. Following this approach, the microstructural features of the diffusion layers obtained under different holding times via vapour-phase type high-temperature low-activity process were experimentally investigated on single crystal CMSX4 alloy. The attention was particularly focused on the effect of the crystallographic orientation of the crystal on the coating features. The evolution of the diffusion layers under different process conditions was then taken into account.
\end{abstract}

\section{Introduction}

CMSX4 alloy is a typical second generation Ni-base single crystal (SC) superalloy characterized by the replacement of most of the $\mathrm{Ti}$ with $\mathrm{Ta}$, by relatively high Co and low Mo content $[1,2]$. The low $\mathrm{Cr}$, high $\mathrm{W}$ and the amount of Re typical of second generation SC alloys (3mass\%), impair the alloy environmental resistance under service conditions. The surface enrichment of Al demonstrated to be particularly helpful for Ni-base alloys similar to that here investigated [3-4]. Among other processes, vapour aluminising, also referred as out-of-pack aluminising, is one of the technique currently available for producing aluminide coating on gas turbine parts. During the process, the aluminium made available by the carrier vapour phase, moves inward into the component by solid state diffusion. In pure $\mathrm{Ni}$, aluminizing results in the formation of a set of single-phase layers, decreasing their $\mathrm{Al}$ content moving away from the surface, is typically found, typically external $\beta$ NiAl layer, intermediate $\gamma^{\prime}-\mathrm{Ni}_{3} \mathrm{Al}$ layer and $\gamma$-Ni substrate. These three phases are also found within the far more complex layered structure of aluminized Ni-base alloys which includes the presence of several single- or multi-phase layers [5].

The first step to investigate the structure of multiphase diffusion layer in a multicomponent alloy is to analyze equilibrium phases and their compositional ranges at different aluminium contents. One simple way to deal with equilibrium conditions in the case of aluminized Ni-base alloys is to separately consider the solubility limits of each alloy element $(\mathrm{X})$ in the Al-Ni-X phase diagram. Such kind of analyses suggests that that the solubility of $\mathrm{Cr}$ in $\beta$-phase decreases as Al concentration increases [6] and the same does the solubility of $\mathrm{W}$ in $\gamma^{\prime}$ [7]. Alternatively, at a given temperature, the partition of elements among phases in equilibrium (i.e. the ratio of the $\mathrm{X}$ content in two phases) gives reason of the driving force for $X$ to cross the interface between two phases in the most convenient direction. A careful analysis of partition of elements between $\beta, \gamma$ and $\gamma^{\prime}$ is given in [5]. 
The effect of an element $\mathrm{X}$ on the stability of these phases is connected to its tendency to form NiAl, $\mathrm{XAl}$ or $\mathrm{Ni}_{3} \mathrm{X}$ compounds. While at $1100^{\circ} \mathrm{C} \mathrm{Cr}$ and $\mathrm{Co}$ were reported to be similarly partitioned in equilibrium among $\beta$ and $\gamma$, they are slightly $\gamma$ rather than $\gamma^{\prime}$ forming. On the other hand $\mathrm{Ta}, \mathrm{Ti}, \mathrm{W}$ and Mo are strongly partitioned in $\gamma^{\prime}$ and less in $\gamma$ rather than in $\beta$ [5]. Apart from the cases of $\mathrm{W}$ and Mo, partition coefficients at a given temperature are substantially composition-independent [5]. Nevertheless, for complex chemical compositions the combined effect of elements should be more deeply considered.

In addition to thermodynamic features, the microstructure of aluminized (and aluminized and serviced) Ni-base alloys is strictly related to kinetic effects, which can induce the presence/absence of equilibrium phases. As a matter of fact the diffusion of alloy elements in the coating phases takes place at different rates due to different interdiffusion coefficients leading to their time- and spatialaccumulations. Typical examples concern the accumulation of the slow diffusing refractory elements, such as $\mathrm{W}$ and Re [1], causing the precipitation of phases, often of non-equilibrium type. Among these, several types of Topologically Close Packed (TCP) phases form, the evolution of which is particularly deleterious during service since they cause local impairment of mechanical strength and they enhance crack initiation [8]. In CMSX4 alloy the presence of $\sigma$ and $\mu$ phases was typically reported [3,9], the latter being also a stable phase for this alloy, relatively rich in Co. During aluminizing processes on the same material, the presence of these phases in different layers depends on the actual process conditions (i.e. temperature, Al surface content, homogenization treatment.) since their formation is related to the local evolution of composition.

Further, the microstructural features of diffusion layered coatings in single crystal are potentially affected by substrate lattice orientation with respect to the external surface. As a matter of fact, solid state diffusion is related by crystallographic orientation of the phase within which it occurs and orientation relationships between lattices in a layered structure exist: (for the present case $<100>_{\gamma} / /$ $<100>_{\gamma}$, while the interface requirement $(111)_{\gamma^{\prime}} / /(110)_{\beta}$ are met by three specific $\beta$ grain orientations with respect to the substrate lattices [4]). The effect of substrate orientation on the overall coating thickness of CM 247 LC alloy was recently investigated in [10]. The authors found that sligth differences in the as-aluminized layer thickness led to significant anisotropy effects of the structure after high-temperature exposition in oxidizing environment. On the other hand the anisotropy of formation, during aluminizing, of TCP phases below the so-called interdiffusion zone was experimentally demonstrated by Murakami et al. on as aluminized TMS-75 alloy [8]. The present paper reports on the preliminary results of an experimental research undertaken to investigate the formation of the layered structure during one step, high temperature aluminizing of CMSX4 alloy and the effects of substrate lattice orientation.

\section{Material and experimental procedures}

A set of discs was cut from round single crystal bars of CMSX4 alloy, a Ni-base alloy nominally containing $9 \% \mathrm{Co}, 6.5 \% \mathrm{Cr}, 6.5 \% \mathrm{Ta}, 6 \% \mathrm{~W}, 5.6 \% \mathrm{Al}, 3 \% \mathrm{Re}, 1 \% \mathrm{Mo}, 1 \% \mathrm{Ti}$ and minor amounts other elements, all expressed in mass \% [2]. The atomic content of the same elements is $9.3 \% \mathrm{Co}, 7.6 \% \mathrm{Cr}$, $2.2 \% \mathrm{Ta}, 2.0 \% \mathrm{~W}, 12.6 \% \mathrm{Al}, 1.0 \% \mathrm{Re}, 0.4 \% \mathrm{Mo}, 1.3 \% \mathrm{Ti}$ [3]. Disc size was $24.5 \mathrm{~mm}$ diameter and 4 $\mathrm{mm}$ thickness. Their longitudinal direction roughly corresponded to [001] direction of the $\gamma$-matrix. After conventional metallographic polishing of the parallel plane surfaces, the discs were aluminised by mean of a low-activity high temperature process at $1050^{\circ} \mathrm{C}$ for different holding times. Once aluminized, each disc was radially cut obtaining two planes. On these sections the layers corresponding to the flat surfaces of discs were oriented normally to [001] direction, while layers intercepted on the cylinder surface by these planes (referred as $45^{\circ}$ and $90^{\circ}$ planes), were normal to [100] and [110] direction, respectively. Metallographic features were investigated on these specimens by means of LOM and SEM observations, these latter combined with EDS microanalyses. Glow Discharge Optical Emission Spectroscopy (GDOES) was also used to supply qualitative chemical profiles along [001] direction. 


\section{Results and discussion}

Microstructural features of coating layers. Typical SEM micrographs obtained by backscattered electrons (BSE) probe is displayed in Fig. 1. Several layers can be distinguished close to the external surface (top-side of the image). To a first approximation, these layers are usually reduced to two layers, in addition to the substrate: the coating ( $\beta$ phase) and the interdiffsion zone (IDZ). In this latter, phases rich in alloying element can be observed together with $\beta$ phase. In addition to these, a mixed zone (MZ) was identified in the present research work (see scheme in Fig.1b) as the layer where these particles appear together with substrate phases ( $\gamma$ and $\left.\gamma^{\prime}\right)$. The additional third layer was differently considered in literature: sometimes it was omitted, including it into IDZ or into substrate $[6,4,11]$, sometimes it was widened to include the whole Al-enriched substrate layer [11] and in other cases it was considered as a secondary reaction zone [8].

When this simple scheme here proposed is chosen, the layers can be detected simply on the basis of light optical and SEM analyses. The coating is typically reported to consist in an external Al-rich layer and in an internal low-Al zone. In the present case the composition of the external surface of the specimen aluminized slightly modified with aluminizing time: for a holding time of $12 \mathrm{~h}$ the $\mathrm{Al}$ was close to $48 \mathrm{at} \%$, in addition to about 2.3 at $\% \mathrm{Co}$ and 1.3 at $\% \mathrm{Cr}$. The internal zone of the coating is characterized by low-Al $\beta$ phase, where substantially only Co and Cr were found among other alloying elements. Close to the interface with IDZ, small Ta-rich particles were detected.

In the IDZ, different layers without evident interfaces can be observed. In each zone, the $\beta$ phase was surrounded by globular particles rich in alloying elements. A detailed analysis of such phases was out of the scope of the present paper, but the punctual analyses of such small-sized particles helped to understand the rather complex chemical profiles of these aluminized samples. Taenrichment (up to 10 times that in the alloy composition) and far less $\mathrm{W}$, Re and Ti enrichments characterized many of the particles in the external part of the IDZ. Several particles mainly containing W, Re, Ta and Cr, roughly corresponding to chemistry of phases reported in [9] were identified at the internal side of the IDZ. Other authors referred the presence of several phases, characterized by the same elements in the outer IDZ [4], so they divided it into sub-layers.

The differentiation between IDZ and MZ was not always clear, depending on the substrate orientation. Within the MZ, the particles rich of refractory elements were still of globular type and they were disposed along parallel lines, separating 'intrusions' of the $\gamma+\gamma$ ' structure from the substrate (Fig. 1a and 1b). At the MZ/substrate interface these particles tended to be thinner and elongated within the $\gamma$ matrix channels, indicating their origin and development from $\gamma$ phase. On both sides close to the interface between MZ and substrate, BSE images showed the enrichment of elements of high atomic weight in $\gamma$ channels close to the substrate. Thus, the MZ/substrate interface corresponds to the position where solubility limits of these elements were exceeded.

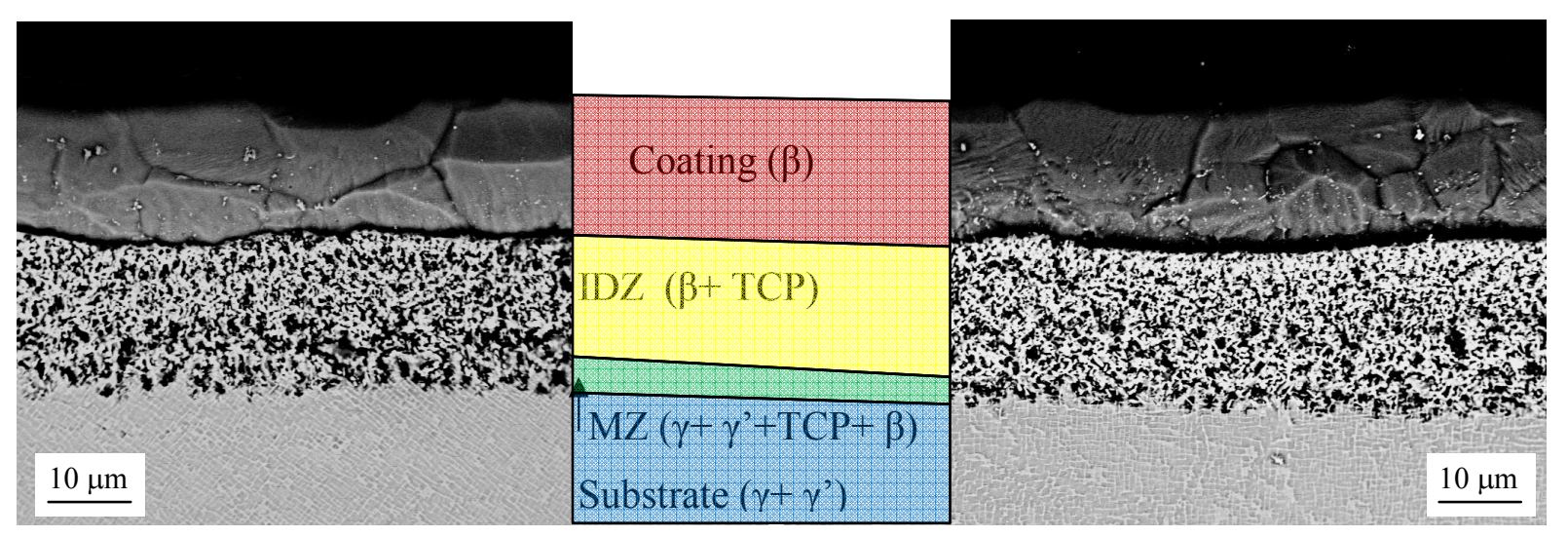

Figure 1. BSE images of the surface layers of the sample aluminized for $6 \mathrm{~h}$ at $1050{ }^{\circ} \mathrm{C}$ in the cases of growth along the [110] and [100] directions of the substrate lattice (left and right respectively). The layers are identified on the micrographs. 

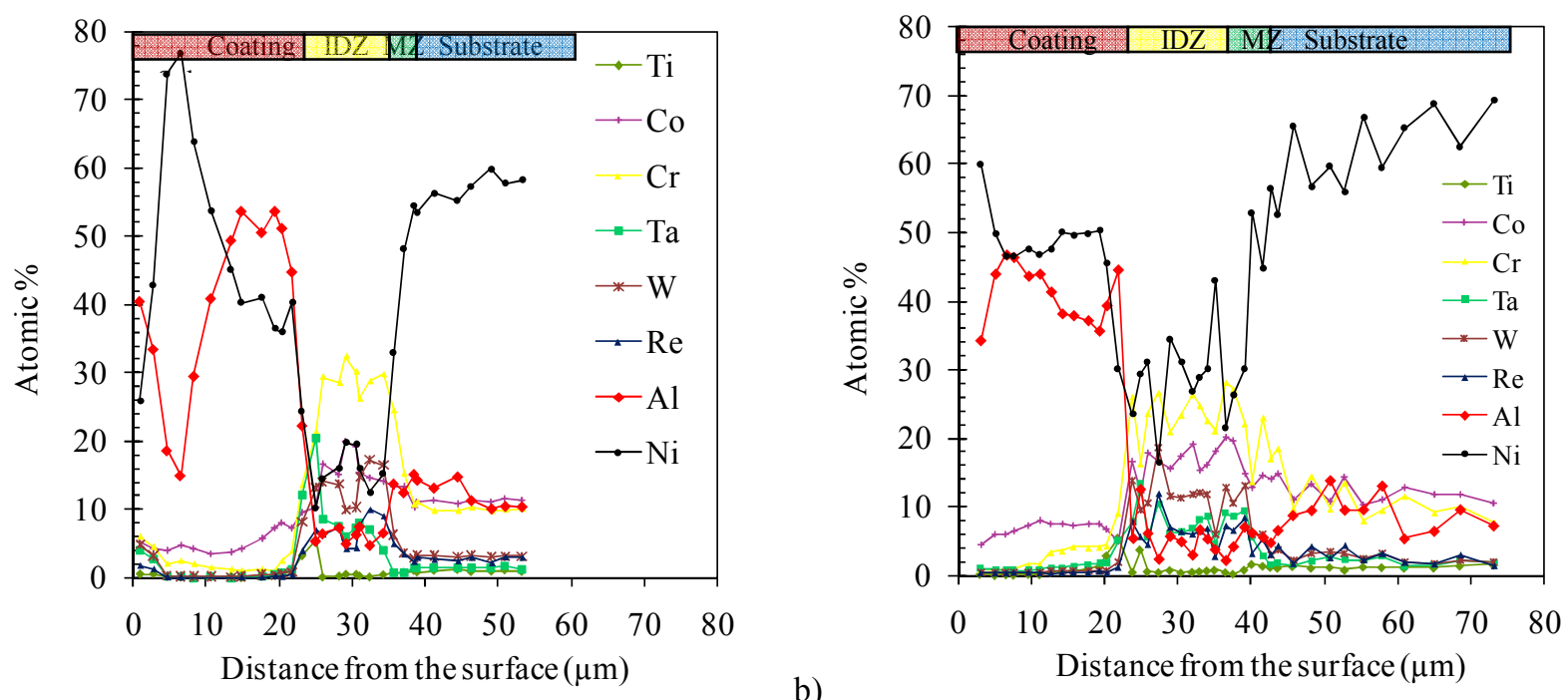

Figure 2. EDS chemical profiles along [001] direction of samples aluminized for $3 \mathrm{~h} \mathrm{(a)} \mathrm{and} 6 \mathrm{~h} \mathrm{(b).}$

Chemical profiles. The chemical profile of the layered structure was obtained by punctual quantitative EDS microanalyses performed along the [001] direction of the samples. Punctual analyses involving a material volume of some $\mu \mathrm{m}^{3}$ resulted in a relatively wide scatter in the nonhomogeneous and fine structures. Nevertheless, EDS profiles (Fig. 2) showed the clear enrichment of some alloying elements in the depth range corresponding to the IDZ and the trends for the main substrate and surface elements: $\mathrm{Ni}$ and $\mathrm{Al}$ respectively.

The chemical profiles supplied by GDOES analyses of aluminized samples were examined in the form here shown in Figure 3. The element content was normalized, i.e. presents as the ratio between the local amount of the specific element to its average content in the substrate. The $x$-axis of the same figures was roughly obtained assuming a constant material removal rate. In such a complex layered structure such GDOES profiles will help to identify the regions of element enrichment/depletion also when their content in the alloy is very low. One of the main features that can be observed in Fig. 3 is that the average composition of the system does not show sudden changes of composition between the IDZ and the substrate, corresponding to the MZ.

Both types of profiles were examined jointly, taking into account the different regions of the aluminized samples. Within the coating, a maximum peak of Al below the surface can be easily seen in both types of profiles and a significant amount of $\mathrm{Co}$ and a slightly lower of $\mathrm{Cr}$ can be found (Fig. 2 and 3a). Further, in the coating, the content of $\mathrm{Cr}$ drastically increases toward the value of IDZ. This features could be related to the fact that, notwithstanding a similar trend for $\mathrm{Co}$ and $\mathrm{Cr}$ to partition in $\beta$ rather in than in $\gamma$ or $\gamma^{\prime}$ [5], Co diffuses in $\beta$ more rapidly than $\mathrm{Cr}$ [6] and this latter is also involved in the formation of particles in the IDZ. A normalized titanium peak corresponding to the position of oxygen, nitrogen and to a small sulfur peaks was observed at about $30 \mathrm{~mm}$ on the $\mathrm{x}$ scale of Fig.3. The presence of nitrides and oxides rich in $\mathrm{Ti}$ is thus supposed to exist. Titanium enrichment was also found in EDS profiles at the external IDZ interface close to the Ta peaks was found. Few micrometer inward, a dramatic local enrichment in carbon can be seen, joined to a small peak of iron, the content of both in the substrate alloy being very limited. Also in this case, the presence and exact location of carbides within the layered structure was not checked during SEM analyses. The presence of oxides, nitrides and carbides could be related to traces of light elements on the surface of the disc at the beginning of aluminum diffusion, these elements formed precipitates once their solubility limits were exceeded.

Moving to the central part of IDZ, after the described peak, the normalized element profile shows evidence of local enrichment in Cr, Mo and W at distance from the surface of about $40 \mu \mathrm{m}$. The enrichment of such elements could correspond to the formation of the phases rich in these elements observed in the IDZ during SEM micrographs. As discussed earlier, the local enrichment is not only 
related to the elemental partition in equilibrium, but it is also a result of elemental diffusion, being it generally slower for $\mathrm{W}$ rather than for Mo and $\mathrm{Cr}$. The presence of Ta and Re was not analyzed (and these elements do not appear in Fig. 3). Nevertheless, a comparison between EDS and GDOES profiles suggests the presence of Re in regions of Cr-, Mo- and W-enrichment. Ta was revealed by EDS profiles to be accumulated in the same IDZ layer, but closer to its external interface in specimens aluminized for short times. A corresponding trend was observed for Ti. Their enrichment extends to the whole IDZ in samples aluminized for longer times. Moving further inside, a wide region with slight $\mathrm{Ni}$ enrichment can be observed in Fig. 4a, corresponding to a slight depletion of refractory elements with respect to their content in the bulk super alloy. A clear identification of this region with respect to the three metallographically-identified layers is not possible. The outward $\mathrm{Ni}$ diffusion and the consequent increased amount of $\gamma^{\prime}$ phase can be observed in the substrate close to the $\mathrm{MZ}$ and within it.
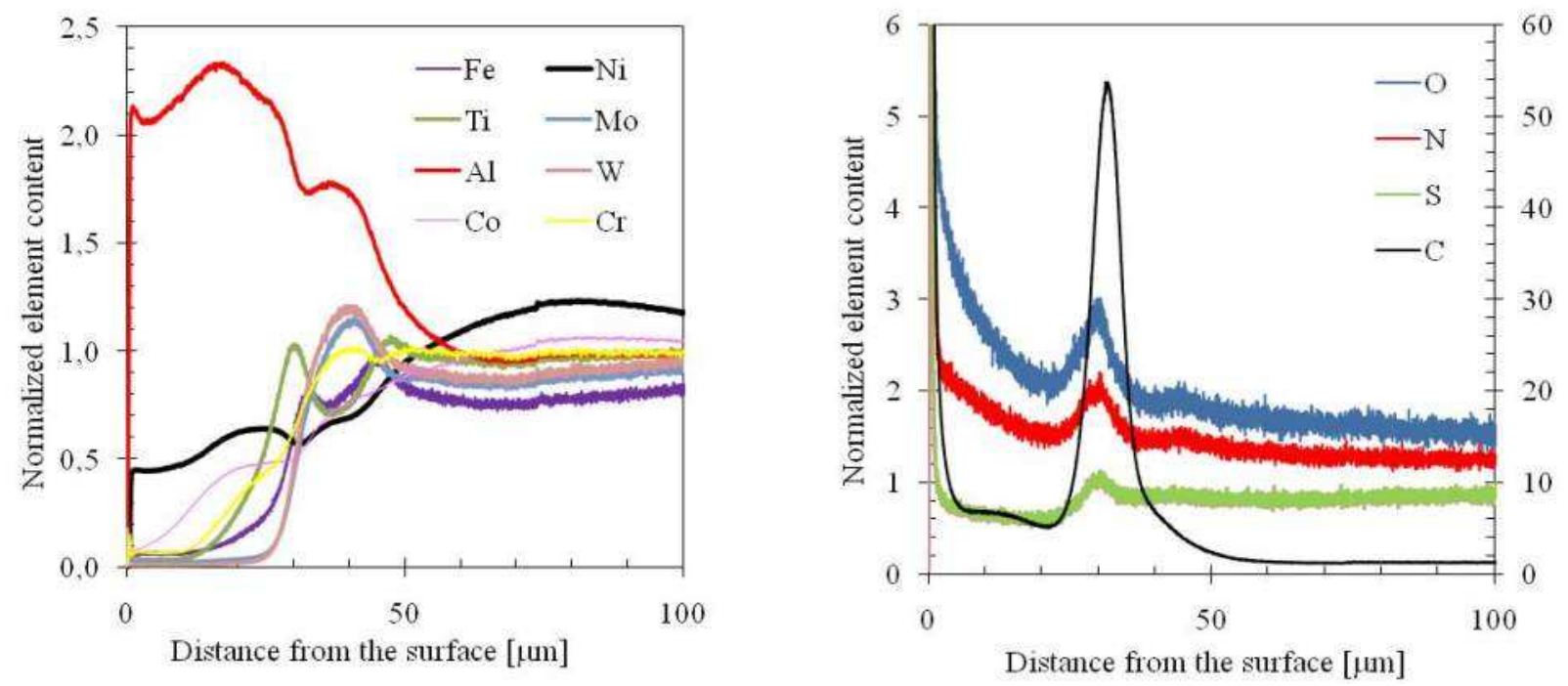

Figure 3. GDOES normalized composition profile normal to the flat surface of the disc aluminized for 6 hours for different elements ([001] direction of the substrate lattice).

Anisotropy of layer thicknesses The orientation-dependence was investigated taking into account the easiest microstructral parameter to be measured, that is the thickness of different layers, here measured on the cylindrical surface of samples. Fig. 4a shows the results for the specimen aluminizd for $6 \mathrm{~h}$; thicknesses of the three layer and of the whole layered structure are plotted as functions of the angular position of the coating where measurements were performed. Anisotropy effects are visible only for IDZ and MZ, this latter being thicker along [110] direction. Nevertheless, as previously mentioned, the thickness of this zone was hardly identifiable for certain microstructural directions, where two sets of parallel structures of particles and $\gamma+\gamma^{6}$ crossed each other. In addition, moving toward the IDZ and within it, such particles coarsen and the possibility to identify their orientation on SEM micrographs was lost. The thickness of the other layers is substantially not affected by the angle formed between the external surface and the reference lattice direction.

Evolution of the layered structure Some differences were observed on compositional profiles of samples aluminized for different times, such as the distribution of elements in the IDZ or in the more homogeneous coating zone. These zones are therefore non-homogeneous and phases within them can change with time. Further, the external coating layer is characterized by a double growth: the inward epitaxial growth of its internal portion and the outward movement of the original surface position [8]. Even keeping in mind the time-dependence of these features, the evolution of the coating was again characterized on the basis of the thickness of the layers. Data along the [110] direction were analyzed, the orientation effects being relatively slight apart from the MZ. Results, displayed in figure $4 \mathrm{~b}$, seem to follow the parabolic law often described in literature. Nevertheless, the limited number of available experimental data prevented the authors from confirming this trend and proposing a reliable thickness-time correlation. 

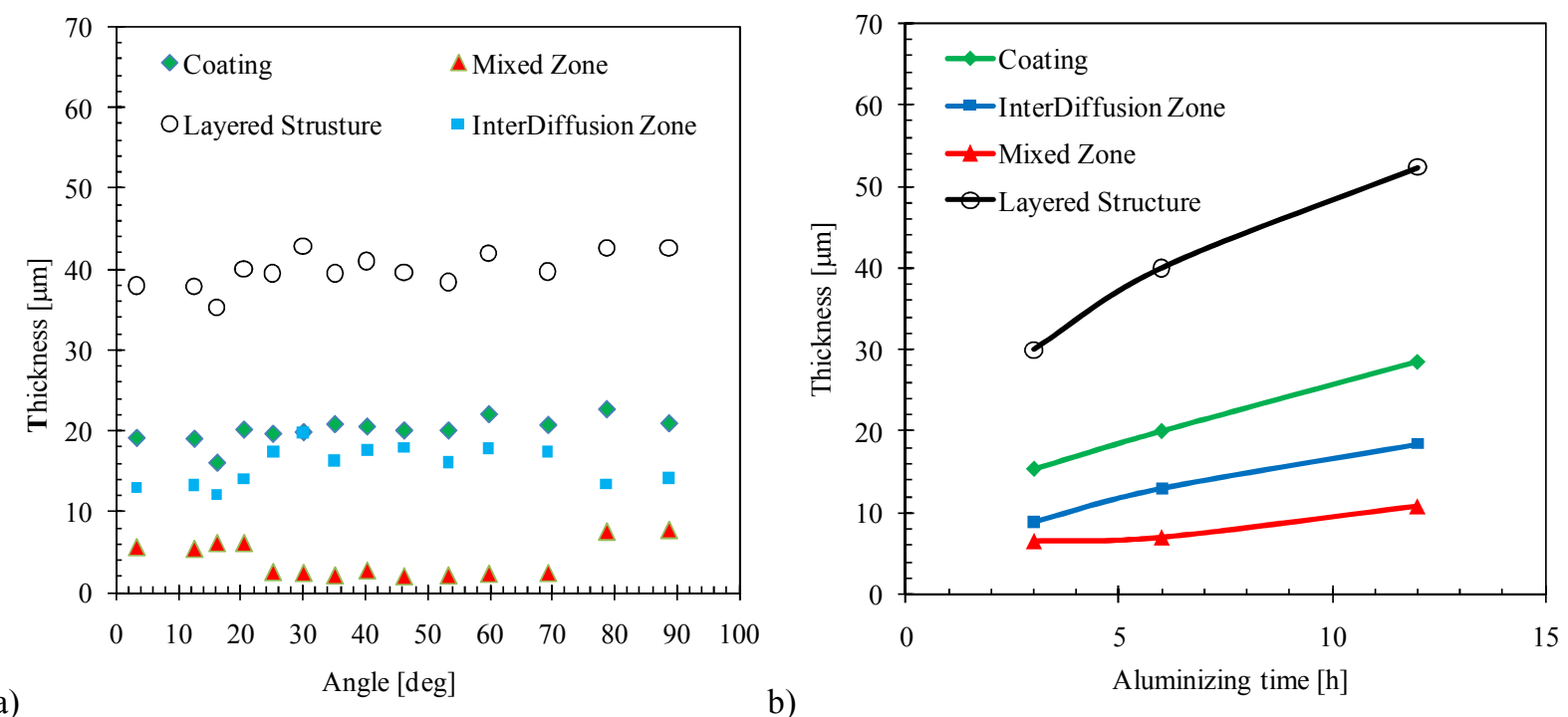

Figure 4. a)Thickness of different layers along the outer cylindrical surface (position identified by $\theta$ angle) in specimen aluminized for $6 \mathrm{~h}$. b)Evolution of the thickness of layers with aluminizing time.

\section{Concluding remarks}

The microstructural features of the layered structure of aluminized of SC CMSX4 alloy were examined taking into account the presence of a mixed zone (MZ) located between this interdiffusion zone (IDZ) and the substrate. The MZ represents a transition region within which refractory elements, rejected by the inward moving $\beta$ phase, accumulate in the $\gamma$ phase channels, and form particles when/where solubility limits are exceeded. Adopting an extremely simplified approach, the microstructural features of the diffusion layers were summarized taking into account their thickness, in all cases increasing with aluminizing time. The anisotropy of the layer structurewas substantially observed only for the MZ, sligthly thicker when growing along [110] direction.

\section{Acknowledgments}

The authors thank P. Bassani, S. Farè, G.M. Vimercati for technical support and helpful discussions.

\section{References}

[1] R.C. Reed: The superalloys: fundamentals and applications. Cambridge Univ. Press. (2006).

[2] M. Durand-Charre: The microstructure of superalloys. Overseas Publishers Association (1997).

[3] C.M.F.Rae, M.S. Hook and R.C. Reed: Mater. Sci. Eng, Vol. A396 (2005), p. 231.

[4] S. Wőllmer, S. Zaefferer, M. Gőken, T. Mack and U. Glatzel: Surf. Coat. Tech., Vol. 167 (2003), p. 83.

[5] C.C. Jia, K. Ishida, and T. Nishizawa: Metall. Mat. Trans., Vol. 25A, (1994), p.473.

[6] H.Wei, H.Y. Zhang, G.C. Hou, X.F. Sun, M.S. Dargusch, X.Yao and Z.Q. Hu: J. Alloys Comp., Vol. 481 (2009), p. 326.

[7] J. Angenete, K. Stiller: Mater. Sci. Eng., Vol. A316 (2001), p. 182.

[8] H. Murakami and T. Sakai: Scripta Mat., Vol. 59 (2008), p.428.

[9] Y.H. Zhang, D.M. Konwles, P.J.Withers: Surf. Coat. Techn., Vol. 107 (1998), p. 76.

[10] F.H. Yuan, Y.S. Yoo, C.Y.Jo, B.G. Choi and Z.Q.Hu: Surf. Coat. Techn., Vol. 183 (2004), p.106.

[11] J. Kohlscheen, H.-R. Stock: Surf. Coat. Techn., Vol.202 (2007), p. 613. 